\title{
UMA PROPOSTA DE USO DA HISTÓRIA DA MATEMÁTICA APOIADA PELAS TIC E HM PARA O ENSINO DE FUNÇÃO
}

\section{PROPOSAL OF THE USO OF MATHEMATICS HISTORY SUPPORTED FOR TIC AND HM TO THE TEACHING OF FUNCTION}

\author{
Luciana Vieira Andrade 1 \\ Universidade Federal do Rio Grande do Norte - UFRN \\ Giselle Costa de Sousa ${ }^{2}$ \\ Universidade Federal do Rio Grande do Norte - UFRN
}

\begin{abstract}
Resumo
Com foco na incorporação da História da Matemática (HM) na sala de aula, este artigo busca apresentar a proposta de uma pesquisa em andamento que pretende estudar a utilização da HM e das Tecnologias da Informação e da Comunicação (TIC) no ensino de conteúdos base do Cálculo Diferencial e Integral (CDI), especificamente a interligação entre a HM e as TIC no processo ensino-aprendizagem das funções. A proposta é apresentar concepções norteadoras do uso das tecnologias em sala de aula, recorrendo a elementos da HM, compondo uma dissertação do Mestrado Profissional do PPGECNM que busca, atendendo às recomendações da CAPES, "ser capaz de construir pontes entre conhecimentos acadêmicos gerados em educação e ensino para sua aplicação em produtos e processos educativos na sociedade" (BRASIL, 2013, p. 01). Para tanto, almeja-se responder à seguinte pergunta-foco: Em que medida a utilização da HM e das TIC é capaz de favorecer a aprendizagem referente às funções? Desse modo, estabelece-se que, tendo como objeto de estudo central as funções matemáticas, tal trabalho irá propor um produto educacional que incluirá uma sequência de atividades apoiadas na HM, com utilização do software Geogebra, que aborde o estudo das funções, buscando verificar em que medida a utilização da HM interligada às TIC contribui para a aprendizagem de funções. Com vistas a alcançar os objetivos descritos, utilizar-se-á uma pesquisa de caráter qualitativo com duas fases distintas: uma teórica, feita com pesquisa bibliográfica e uma fase prática, pautada na pesquisa ação, contemplando a investigação matemática (IM).
\end{abstract}

Palavras-chave: Matemática; História; Tecnologia; Ensino; Função.

\section{Abstract}

Focusing on the incorporation of the History of Mathematics (HM) in the classroom, this article aims to present the proposal for an ongoing study that aims to study the use of the HM and Information and Communication Technologies (TIC) in the basic content of education Differential and Integral Calculus (CDI), specifically the link

\footnotetext{
${ }^{1}$ luvieira13@hotmail.com.

2 giselle@ccet.ufrn.br.
} 
between HM and TIC in the teaching-learning process functions. The proposal is to present guiding conceptions of the use of technology in the classroom, using elements of HM, composing a dissertation PPGECNM Professional Master seeking, given the CAPES recommendations, "ser capaz de construir pontes entre conhecimentos acadêmicos gerados em educação e ensino para sua aplicação em produtos e processos educativos na sociedade" (BRAZIL, 2013, p. 01). Therefore, we aim to answer the question Focus: To what extent the use of the HM and TIC is able to promote the learning related to functions? Thus, it is established that, with the central object of study mathematical functions, such work will propose an educational product that will include a series of activities supported in HM, using the Geogebra software, which addresses the study of functions, in order to verify the extent to which the use of HM interconnected TIC contributes to learning functions. In order to achieve the objectives described, will be used, a qualitative research with two distinct phases: a theoretical, made with literature and a practical phase, based on action research, covering mathematics research (IM).

Keywords: Mathematics; History; Tecnology; Teaching; Fucntion.

\section{Introdução}

Com vistas a apresentar concepções norteadoras do uso das tecnologias em sala de aula recorrendo a elementos da História da Matemática, este artigo procura apresentar a proposta de uma pesquisa em andamento que busca obter elementos científicos que irão culminar com a dissertação referente à finalização do Mestrado Profissional do PPGECNM. Este programa faz parte, conforme o Documento de Área 2013 da Coordenação de Aperfeiçoamento de Pessoal de Nível Superior (CAPES), daqueles que desenvolvem pesquisas na área de Ensino, que se insere na Grande Área Multidisciplinar, e tem como foco estudos e produtos referentes ao Ensino de Ciências e Matemática. Segundo este mesmo documento, "busca interlocução com as áreas geradoras dos conteúdos a serem ensinados e tem foco na integração entre conteúdo disciplinar e conhecimento pedagógico" (BRASIL, 2013, p. 01) e ainda "tem como objeto a mediação do conhecimento em espaços formais e não formais de ensino e como principal objetivo, a construção de conhecimento científico sobre este processo e sobre fatores de caráter micro e macro estrutural que nele interferem" (BRASIL, 2013, p. 01).

Além disso, como dentre as linhas de pesquisas vinculadas a tal programa, esta se enquadra na linha de História e Filosofia da Ciência e Matemática. Por isso, o presente trabalho focaliza a incorporação da História da Matemática (HM) na sala de aula a partir do estudo e utilização da HM e das Tecnologias da Informação e da Comunicação (TIC) no ensino de conteúdos base do Cálculo Diferencial e Integral 
(CDI), especificamente a interligação entre a $\mathrm{HM}$ e as TIC no processo ensinoaprendizagem das funções.

Deste modo, a partir dessas informações, pretende-se desenvolver uma pesquisa que atenda a tais orientações e ainda que "seja capaz de construir pontes entre conhecimentos acadêmicos gerados em educação e ensino para sua aplicação em produtos e processos educativos na sociedade" (BRASIL, 2013, p. 01). Para isso, pretende-se desenvolver um produto educacional que incluirá uma sequência de atividades, especialmente com utilização da HM apoiada por recursos tecnológicos (software de matemática dinâmica: Geogebra) que aborde os elementos constituintes de uma função matemática e suas diferentes formas de representação. Conceito este unificador de vários outros e base para o CDI.

Estreitando-se as relações com a pesquisa científica desenvolvida nesta área, busca-se responder à seguinte pergunta-chave: Em que medida a utilização da HM e das TIC é capaz de favorecer a aprendizagem referente às funções?

Para responder a pergunta-foco, algumas intenções foram estabelecidas. Como objetivo geral a que se pretende alcançar, tem-se: verificar em que medida a articulação de HM e TIC em sala de aula, favorece a aprendizagem quanto ao estudo das funções. Como desmembramento, surgem os seguintes objetivos específicos: averiguar se existe, na prática docente, a utilização da HM e das TIC como recurso didático, isoladamente e/ou conectados; realizar levantamento bibliográfico sobre história das funções para selecionar problemas e/ou episódios a serem tratados pelas TIC; analisar as potencialidades de interligação entre a HM e as TIC em busca da melhoria no ensino das funções e utilizá-las como apoio para seu aprendizado; elaborar sequência de atividades relacionando HM e TIC para o ensino das funções; testar e refinar o produto educacional.

Tendo em vista essas ideias centrais, seguem os itens que fundamentam teoricamente este artigo e a pesquisa supracitada.

\section{Fundamentação Teórica}

O referencial teórico desse artigo apresenta duas categorias que respaldam a pesquisa nos aspectos de conteúdo, práticos, metodológicos e científicos. Cada uma delas relaciona três tópicos que abordam os itens considerados imprescindíveis quanto à fundamentação que será utilizada como apoio em todas as etapas de desenvolvimento da 
pesquisa e deixam claras as referências que serão utilizadas no desenrolar de todo o trabalho.

Recomendações de Documentos Oficiais: PCN, BNC e PNLD para o Ensino de Funções, uso de HM e TIC

Documentos como os Parâmetros Curriculares Nacionais para o Ensino Médio (PCNEM) e a Base Nacional Comum Curricular (BNC), que direcionam e organizam o aprendizado, ou seja, sinalizam percursos de aprendizagem e desenvolvimento dos estudantes ao longo da EB, consideram importante a busca pela interdisciplinaridade e contextualização para "produzir um conhecimento efetivo, de significado próprio e cujo aprendizado seja parte essencial da formação cidadã” (BRASIL, 2000, p.04). Interdisciplinaridade essa que não cancela a disciplinaridade do conhecimento (BRASIL, 2000).

Atribuir sentido aos conceitos é uma abordagem tratada no BNC. Nessa ideia aparece a importância da utilização do recurso contextualização, estratégia capaz de contribuir para a busca por atingir os objetivos gerais da Matemática na EB: "estabelecer conexões entre os eixos da Matemática e entre essa e outras áreas do saber e comunicar-se, utilizando as diversas formas de linguagem empregadas em Matemática" (BRASIL, 2015, p.118), bem como evidenciar a relevância social e cultural da área e seu papel no desenvolvimento histórico da ciência (BRASIL, 2015). Nessa perspectiva, faz-se jus ao uso da HM como tendência atual da Educação Matemática e via de ligação para assunto integrador como função. De fato, a presença dessa tendência e assunto aparece ainda na apresentação dos objetivos gerais da Matemática no EM, os quais consistem, conforme a BNC, em:

\footnotetext{
Analisar criticamente os usos da Matemática em diferentes práticas sociais e fenômenos naturais, para atuar e intervir na sociedade e recorrer às tecnologias digitais para descrever e representar matematicamente situações e fenômenos da realidade, em especial aqueles relacionados ao mundo do trabalho (BRASIL, 2015, p.142).
}

Nesse mesmo contexto, os PCNEM explicitam as competências e habilidades referentes à aprendizagem da Matemática, que consideram o relacionamento da Matemática com outras áreas tendo em vista sua relação estreita com o todo social e cultural, isto é, sua dimensão histórica. Dentre as competências apresentadas, 
destacaremos aqui a contextualização sócio-cultural (BRASIL, 2000) que, em relação ao uso da história das ciências, recomenda um trabalho direcionado aos itens:

Reconhecer o sentido histórico da ciência e da tecnologia, percebendo seu papel na vida humana em diferentes épocas e na capacidade humana de transformar o meio e compreender as ciências como construções humanas, entender que se desenvolveram por acumulação, continuidade ou ruptura de paradigmas, relacionando o desenvolvimento científico com a transformação da sociedade (BRASIL, 2000, p.13).

Especificamente em relação à Matemática, a competência aparece da seguinte forma: "relacionar etapas da história da Matemática com a evolução da humanidade" (BRASIL, 2000, p.46). Por conseguinte, é nesta perspectiva que recorreremos a HM para tratar o assunto Função via TIC.

Sob o ponto de vista da análise social e histórica, orientam:

O currículo do EM deve garantir também espaço para que os alunos possam estender e aprofundar seus conhecimentos sobre números e álgebra, mas não isoladamente de outros conceitos, nem em separado dos problemas e da perspectiva sócio-histórica que está na origem desses temas (BRASIL, 2000, p.44).

Essa sugestão parte do princípio de que o aluno deve "perceber o valor da Matemática como bem cultural de leitura e interpretação da realidade e possa estar melhor preparado para sua inserção no mundo" (BRASIL, 2000, p.45). Além disso, abre margem ao uso de Função em suas diferentes representações como gráfica, numérica e algébrica, articuladas e não isoladamente.

Para tanto, há a necessidade de se adotarem métodos de aprendizado ativo e interativo, sendo a utilização da HM um importante recurso, conforme descrevem:

A compreensão da relação entre o aprendizado científico, matemático e das tecnologias e as questões de alcance social são a um só tempo meio para o ensino e objetivo da educação. Isso pode ser desenvolvido em atividades como os projetos, ou se analisando historicamente o processo de desenvolvimento das Ciências e da Matemática. Nessa medida, a história das Ciências é um importante recurso. A importância da história das Ciências e da Matemática, contudo, tem uma relevância para o aprendizado que transcende a relação social, pois ilustra também o desenvolvimento e a evolução dos conceitos a serem aprendidos (BRASIL, 2000, p.54). 
O Programa Nacional do Livro Didático (PNLD) considera como princípio de avaliação de Matemática, a ideia de que ela foi "produzida e organizada no decorrer da história" e ainda que é "uma das mais significativas conquistas do conhecimento humano" (BRASIL, 2015, p.11). No item contextualização, o guia pondera que:

No intuito de avaliar ligações entre conteúdos matemáticos e práticas sociais
de hoje são analisadas as conexões com outros campos do saber. Avaliam-se,
ainda, as contextualizações feitas com base na HM, com o objetivo de tornar
o estudo mais significativo. Focaliza-se, também, em que medida na obra,
são propostos temas e atividades que incentivam o desenvolvimento de
posturas e de valores importantes para o exercício da cidadania (BRASIL,
2015, p.18).

$\mathrm{Na}$ ficha de avaliação do LD, no critério metodologia de ensino e aprendizagem, julga-se se: "os conhecimentos matemáticos são contextualizados, de forma significativa, no que diz respeito, entre outras questões, a práticas sociais atuais e à HM" (BRASIL, 2015, p.78). Logo, mais uma vez, justifica-se o desenvolvimento de produto educacional com base no uso da HM em favor do ensino. Para tanto, buscam-se informações que sejam além da simples identificação de datas, locais e personagens considerados gênios percebendo se há "atribuição de significado aos conteúdos tanto a partir da evolução histórica dos conceitos e de suas inter-relações, quanto das motivações sociais, econômicas e científicas que levaram ao avanço da Matemática" (BRASIL, 2015, p.106). Nesta óptica, fazemos referência e uso da história significado de Miguel (1993) e a entendemos como fonte de seleção de episódios e métodos adequados ao ensino, particularmente, de função como elemento integrador da educação básica e ensino superior, bem como, de assuntos do CDI.

Concordando com as ideias que aparecem nos documentos oficiais, buscaremos utilizar a HM para dar significado ao ensino da Matemática, por meio de sua articulação com as TIC, entendendo que estratégias inovadoras favorecem o aprendizado. Essa interligação ocorrerá tanto na etapa de elaboração do produto educacional quanto nos momentos de aplicação dessas atividades bem como quando, de posse dos dados coletados, faremos a análise qualitativa das informações.

Realmente, os mesmos documentos apontam que o uso de recursos da tecnologia no EM enriquece o ensino da Matemática. Utilizar softwares, sites de pesquisa, planilhas eletrônicas ou calculadora, de maneira ética, auxilia a aprendizagem e contribui para a formação do aluno como cidadão completo e preparado para o mercado 
de trabalho. Além disso, como posto, recomendam constantemente o ato investigativo via História. (BRASIL, 2014; 2010).

Sobre a HM, as TIC e a IM

Visando motivar as práticas investigativas em sala de aula, articulações entre HM, TIC e IM exercem grande fascínio, sobretudo, na Educação Matemática (EM). São práticas inerentes à formação de professores as investigações científicas, cujos elementos essenciais são a reflexão, a atuação e a sistematização. Para Garnica e Souza (2013), a EM é uma área voltada à compreensão da Matemática no contexto de ensino e aprendizagem, cabendo a ela estudar como, no tempo, se têm desenvolvido as produções as alterações e as manutenções de ensino da Matemática.

Miguel e Miorim (2011) argumentam que a HM investiga todas as dimensões da atividade matemática na história das práticas sociais envolvidas no processo de produção de seu conhecimento. Sua marca registrada é manter o verdadeiro diálogo entre as duas áreas: História e Matemática, com vistas à compreensão das alterações e permanências nas práticas educativas ligadas à produção do conhecimento dessa ciência. Os mesmos autores ainda informam que "a história pode ser fonte de busca de compreensão e de significados para o ensino-aprendizagem da Matemática escolar na atualidade" (MIGUEL; MIORIM, 2011, p. 45) e julgam ainda que cabe ao professor fazer a história participar do processo de ensino, intencionalmente. É nesta perspectiva que usamos a HM em nossa proposta.

Considerando a utilização de problemas históricos como mais um elemento motivador para o ensino, Miguel e Miorim (2011, p. 52) defendem a ideia de que ela também tem como atribuição o "desenvolvimento de atitudes e valores favoráveis" bem como a "compreensão das relações entre tecnologia e herança", desmistificando o pensamento de que a Matemática é uma área que já está pronta e acabada. Por este motivo, creditamos na possibilidade da HM ser potencializada pelas TIC e IM numa aliança em prol do ensino e aprendizagem da Matemática que passa por um processo de fazer matemático investigativo, reflexivo e significante em que o aluno encontra justificativas aos seus porquês, vê que pode produzir também matemática e reflete sobre suas descobertas. Assim, nos apoiamos na forma de participação da história na educação como aquela que ela se responsabiliza por mostrar a maneira de produção do conhecimento ao invés de dedicar-se exclusivamente a destacar apenas os resultados, como defendem Miguel e Miorim (2011). 
Com relação ao uso da HM, Miguel e Miorim (2011) sugerem também que ela cumpra o papel didático-metodológico, ideia que nos apoiaremos já que nossa intenção é desenvolver um trabalho que investigue o conceito de funções no decorrer do tempo e ainda problematize de maneira investigativa suas diferentes formas de representações, considerando "problematização também é um método" (MIGUEL; MIORIM, 2011, p. $155)$.

Entendendo que o movimento histórico não ocorre de modo contínuo nem, tampouco, linearmente, cabe, nessa proposta, destacar como ocorreu o pensamento e a formação do conhecimento matemático com o decorrer do tempo. Dessa maneira, a HM contribuirá para a aplicação de estratégias como interdisciplinaridade e contextualização no ensino. De fato, ao estudar a história das Funções, percebe-se sua articulação com diferentes áreas e contextos, o que pode inspirar em nossas atividades, diferentes abordagens nesta perspectiva.

$\mathrm{Na}$ sociedade e no mundo educacional a tecnologia de informação e comunicação se faz cada vez mais presente. Alguns programas de computador voltados para o ensino e aprendizado da Matemática nos permitem dispor de alguns recursos que, usados de forma adequada, se convertem em ferramentas potentes e com enorme funcionalidade. Nessa perspectiva, Néri (2010) destaca que:

O uso de tecnologia para o ensino da matemática, amparado por um software, pode ter um papel decisivo nas expectativas do professor em ensinar aos alunos alguns conceitos que, da forma tradicional poderiam apresentar resultados não muito interessantes no ponto de vista pedagógico (NÉRI, 2010, p. 01).

Realmente, os softwares educacionais tem apresentado papel importante na vida dos jovens atuais tendo em vista que já são frequentes no seu cotidiano. Logo, têm conquistado espaço na vida dos estudantes, por apresentarem diversas possibilidades de exploração, análises e manuseio, além de permitir os alunos a programarem e modelarem problemas do cotidiano. Essas análises facilitam os processos de construção do conhecimento, pois possibilitam uma relação de interação do aluno com o recurso computacional. De fato,

O computador pode ser um importante recurso para promover a passagem da informação ao usuário ou facilitar o processo de construção de conhecimento. No entanto, por intermédio da análise dos softwares, é possível entender que 
o aprender (memorização ou construção de conhecimento) não deve estar restrito ao software, mas à interação do aluno-software (VALENTE,1999, p.52).

Assim sendo, o uso adequado de tais recursos implica na interação do aluno com o mesmo, em favor do desenvolvimento do conhecimento e como coletivo pensante que Borba (2001) denomina por coletivos-seres-humanos-com-mídia, e não na perspectiva de que a máquina/mídia supera tal ação, ou seja, pensa pelo aluno.

Como cita Valente (1999, p. 11):

\footnotetext{
Por outro lado, se esses novos softwares ampliam as possibilidades que o professor dispõe para o uso do computador na construção do conhecimento, também demandam um discernimento maior por parte do professor e, consequentemente, uma formação mais sólida e mais ampla.
}

Portanto, o uso dos computadores, para fins didáticos pedagógicos, é algo que possibilita ao professor enriquecer suas práticas educacionais, permitindo-o realizar simulações, exercícios e até comprovando propriedades que dificultam o entendimento do aluno, no entanto, além da formação sólida que o educador deve ter, deve também ter discernimento para superar as dificuldades encontradas no espaço escolar.

A proposta do trabalho com investigação está intimamente ligada ao estudo de questões, não necessariamente muito sofisticadas, que permitam a construção do conhecimento por meio da formulação de conjecturas (PONTE; BROCARDO; OLIVEIRA, 2013).

Em Matemática, investigar tem características peculiares que, para Ponte, Brocardo e Oliveira (2013), estão mais fortemente representadas pelas ideias da tríade: conjectura-teste-demonstração.

Em busca de informações sobre o que é desconhecido, os matemáticos vislumbram a investigação como a descoberta das relações entre objetos matemáticos com vistas à identificação de suas propriedades. Com essa finalidade, Ponte, Brocardo e Oliveira (2013) argumentam que a Investigação Matemática (IM) envolve quatro etapas: Reconhecimento da situação, sua exploração e a formulação de questões; Formulação de conjecturas; Realização de testes e refinamento das conjecturas; Argumentação, demonstração e avaliação do trabalho realizado. No desenrolar da pesquisa, faremos uso das afirmações defendidas por Ponte, Brocardo e Oliveira (2013), quanto à IM, nos momentos da elaboração e das aplicações das atividades tendo em 
vista que o uso da HM pode colocar o aluno no papel do matemático e, portanto, investigador/pesquisador, que produz conhecimento, apoiado pelas TIC.

\section{Percurso Metodológico}

Com vistas a alcançar os objetivos já estabelecidos aqui, utilizar-se-á uma pesquisa de caráter qualitativo já que há a concordância com as ideias de Ludke e André (1986) no que diz respeito às suas principais características: as fontes são ambiente natural; os dados predominantemente descritivos; interessa-se mais pelo processo do que pelo produto; foco do pesquisador são os significados atribuídos pelos envolvidos às coisas e à vida; além de realizar análise indutiva dos dados.

Cabe aqui descrever que a pesquisa ocorrerá por intermédio de duas fases distintas: uma fase teórica, feita por meio de pesquisa do tipo bibliográfica definida, por Gil (2008, p. 16) como sendo "desenvolvida com base em material já elaborado, constituído principalmente de livros e artigos científicos", onde serão estudados os elementos que fundamentarão o trabalho por meio de leituras de livros, teses, dissertações, artigos em periódicos nacionais e estrangeiros, ensaios e anais de congressos. A fase seguinte terá uma natureza mais prática caracterizando-se pela perspectiva da pesquisa ação. Deste modo, irá contemplar a investigação matemática a partir da observação e análise de dados coletados em entrevistas (transcritas), observações de aulas (diário de campo) e realização de cursos de extensão para alunos e professores. Com vistas à análise de documentos, também serão úteis instrumentos como registros fotográficos e de vídeo, livros, questionários, atividades de alunos e relatórios de observação. De modo geral, serão organizados em tabelas, gráficos, imagens e textos transcritos.

A escolha pelo tipo pesquisa-ação se deu pela concordância com as ideias de Lewin (apud LUDKE e ANDRÉ, 1986, p. 31) no que concerne “à pretensão de se investigar as relações sociais e conseguir mudanças em atitudes e comportamentos dos indivíduos". Segundo Ludke e André (1986):

Com a denominação de investigação-ação (action research), os livros de pesquisa da década de 1950 descrevem essa metodologia como uma ação sistemática e controlada desenvolvida pelo próprio pesquisador. Um exemplo clássico é o professor que decide fazer mudança em sua prática docente e a acompanha com um processo de pesquisa, ou seja, com um planejamento de 
intervenção, coleta sistemática de dados, análise fundamentada na literatura pertinente e relato dos resultados (LUDKE; ANDRÉ, 1986, p.31).

Os elementos e as informações apresentadas na fundamentação teórica desse artigo compõem a proposta de trabalho em andamento. Para a fase inicial, a teórica, está sendo realizada uma pesquisa bibliográfica acerca do conceito de função, seus elementos e formas de representação em diferentes épocas a fim de se identificar possíveis alterações/aplicações que possam ter ocorrido com o passar do tempo. Nessa etapa, a HM é usada como fonte de seleção de episódios e métodos, bem como, aparece como recurso que contribuirá para se atribuir significado ao ensino a partir da evolução histórica de conceitos, como propõe a BNC. Como resultado parcial, tem se chegado que o conceito de função é forte aliado ao ensino da Matemática e de outras áreas do conhecimento por permitir a descrição do comportamento de fenômenos no cotidiano através da leitura, interpretação e construção dos gráficos (BRASIL, 2000). De fato,

\begin{abstract}
Para alguns pesquisadores a noção de dependência teve inicio há cerca de 6000 anos, porém foi somente nos três últimos séculos que houve o desenvolvimento do conceito formal de função, com estreita ligação com problemas relacionados ao Cálculo e à Análise. O instinto de funcionalidade surgiu da necessidade do Homem, levado pela premência de fazer associações entre os objetos. Como exemplo, podemos citar os pastores da antiguidade que necessitavam 'contar' suas ovelhas, sem ainda disporem de um sistema de contagem desenvolvido. Para resolver o problema eles associavam a cada ovelha uma pedra o que permitia a conferência do rebanho que retornava da pastagem ao final do dia. De forma mais sistemática percebe-se a idéia de função entre os babilônios que construíram tabelas em argila onde para cada valor na primeira coluna existia um número correspondente na segunda. (HISTÓRIA ..., 2015)
\end{abstract}

Levando isto em consideração, posteriormente, a ideia é elaborar e aplicar atividades sobre Função que permitam a utilização do Geogebra articulado a HM e IM, atendendo ao que recomendam os documentos oficiais em relação ao uso desses recursos para atingir aos objetivos do ensino da Matemática na EB.

Em nossos estudos da aliança entre HM e TIC chegamos à conclusão que tais atividades devem respeitar as seguintes fases: Escolha do conceito matemático/Tópico/Episódio a ser ensinado/estudado; Coleta de informações históricas sobre sua criação, o contexto social, histórico, econômico, artístico, entre outros aspectos que permearam à época da criação, reconhecendo, assim, uma situação problemática, vista por Ponte, Brocado e Oliveira (2013) como a primeira etapa da IM; Uso das informações históricas como um processo de recriação investigativo dos 
acontecimentos que levaram as conclusões, fórmulas e/ou conceitos matemáticos, relacionando-a, sempre que possível, com o contexto atual dos estudantes; Emprego das TIC para otimizar o tempo das (re)criações, aproveitado no auxílio das investigações, reflexões e comparações do que foi encontrado pelos estudantes e os resultados históricos conhecidos, conduzindo os estudantes às últimas etapas da IM de testar, reformular e validar as conjecturas; Por fim, a socialização dos resultados e formalização em relatórios.

Deste modo, as atividades históricas articuladas com TIC devem ser compostas pelos seguintes elementos: título, objetivos, materiais, conhecimentos prévios, recortes e informações históricas, procedimentos/passos (usando TIC), momentos de reflexão, relatório e discussões.

\section{Considerações finais}

As leituras realizadas para produção desse artigo proporcionaram conhecimentos quanto aos principais elementos referentes à utilização da HM e das TIC, com apoio da IM, no ensino da Matemática, particularmente, sobre Função. Pela reflexão, estabelecemos que a utilização de tais recursos em sala de aula de maneira fundamentada enriquecerá a prática docente, de maneira tal que as atividades elaboradas e aplicadas buscarão, com muita proximidade, atender ao que orientam os documentos oficiais bem como às ideias defendidas no referencial teórico. Logo, chegamos às bases que fundamentam a proposta de incorporação da HM, a partir do conceito de Função, na sala de aula apoiada pelas TIC e IM apontando os caminhos percorridos e a seguir.

A aplicação da abordagem da HM de forma crítica e utilização das TIC permitem o aperfeiçoamento da prática pedagógica, colaborando com a melhoria nos processos de ensino e aprendizagem. Concluímos então que pensar a Matemática da escola com uma visão mais global propiciada pela HM viabiliza o trabalho via TIC, particularmente, para abordagem do conceito de Função.

\section{Referências}

BRASIL. Base Nacional Comum Curricular. Brasília: Ministério da Educação, Secretaria de Educação Básica, 2014. 302p.

BRASIL. CAPES. DOCUMENTO DE ÁREA: Avaliação Trienal 2013. Disponível: 
https://docs.google.com/viewer?a=v\&pid=sites\&srcid=Y2FwZXMuZ292LmJyfHRyaW VuYWwtMjAxM3xneDoxY2FmZGFjZGIyNzE3Nzlh. Acesso em 14/12/2015.

BRASIL. Guia de livros didáticos: PNLD 2015 - Matemática Ensino Médio. Brasília: Ministério da Educação, Secretaria de Educação Básica, 2014. 108p.

BRASIL. Parâmetros Curriculares Nacionais Ensino Médio: Ciências da Natureza, Matemática e suas Tecnologias. Brasília: Ministério da Educação, Secretaria do Ensino Médio, 2000. 58p.

BORBA, Marcelo de Carvalho. Coletivos seres-humanos-com-mídias e a produção do conhecimento matemático. In: I Simpósio Brasileiro de Psicologia da Educação Matemática, 2002, Curitiba. I Simpósio Brasileiro de Psicologia da Educação Matemática, 2001. v. 1. p. 135-146. Disponível em: $<$ http://www.rc.unesp.br/gpimem/downloads/artigos/borba/borba_coletivos-sereshumanos-com-midias.pdf>.

GARNICA, A. V. M.; SOUZA, L. A.. Educação Matemática, História, História da Matemática e História da Educação Matemática. In: Elementos de história da educação matemática. São Paulo: Coleção Cultura Acadêmica - Editora UNESP, 2013. . pp. 17-48. Disponível em:

http://www.culturaacademica.com.br/_img/arquivos/Elementos_de_historia_da_ed_mat -WEB.pdf . Acesso em 14/12/2015.

GIL, A. C. Como elaborar projetos de pesquisa. 4. Ed. São Paulo: Atlas, 2008.

HISTÓRIA da função. 2015. Disponível em: http://www.unifalmg.edu.br/matematica/?q=hist_funcao. Acesso em: 05 dez. 2015.

LUDKE, M., ANDRÉ, M. E. D. A. Pesquisa em educação: abordagens qualitativas. São Paulo: EPU, 1986.

MIGUEL, A. Três estudos sobre História e Educação Matemática. 1993. 361f. Tese (Doutorado em Educação) - Faculdade de Educação, Universidade de Campinas, Campinas, 1993.

MIGUEL, A.; MIORIM, M. A.; História na Educação Matemática: proposta e desafios. 2 ed. .- Belo Horizonte: Autêntica Editora, 2011.

NÉRI, I.C. Funções Polinomiais do Segundo Grau Mediados pelo Software Geogebra na Perspectiva dos Registros de Representação Semiótica, São Paulo, 2010. Disponível em:

http://www.sinprosp.org.br/congresso_matematica/revendo/dados/files/textos/Relatos/F UN\%C3\%87\%C3\%95ES\%20POLINOMIAIS\%20DO\%20SEGUNDO\%20GRAU\%20 MEDIADOS\%20PELO\%20SOFTWARE\%20G.pdf. Acesso em14/12/2015.

PONTE, J. P. da, BROCARDO, J., OLIVEIRA, H. Investigações matemáticas na sala de aula. 3 ed. Ver. Ampl.- Belo Horizonte: Autêntica Editora, 2013. 
Luciana Vieira Andrade e Giselle Costa de Sousa

Uma proposta de uso da história da matemática apoiada pelas TIC e HM para o ensino de função

VALENTE, J. A. (Org). O computador na sociedade do conhecimento. 1 ed.

UNICAMP / NIED, Campinas, 1999. 